\title{
INTERNET OF THINGS IN THE CONTEXT OF INDUSTRY 4.0: AN OVERVIEW
}

\author{
Georgios Lampropoulos \\ Department of Informatics, Alexander Technological Educational Institute of Thessaloniki \\ Thessaloniki, Greece \\ lamprop.geo@gmail.com
}

\section{Kerstin Siakas}

Department of Informatics, Alexander Technological Educational Institute of Thessaloniki Thessaloniki, Greece

siaka@it.teithe.gr

\section{Theofylaktos Anastasiadis}

Department of Physical Education and Sport Sciences, Aristotle University of Thessaloniki Thessaloniki, Greece

anastheo@phed.auth.gr

\begin{abstract}
Due to successive technological advancements, developments and innovations, the global industrial landscape has drastically transformed over the last years. The fourth industrial revolution (Industry 4.0) aims at transforming traditional industries into intelligent ones by incorporating innovative technologies. Industry 4.0 enables physical assets to be integrated into intertwined digital and physical processes thus creating smart factories and intelligent manufacturing environments. Internet of Things (IoT) is a rapidly growing technology that has drastically contributed to the Industry 4.0 realization. IoT pursues to pervade our everyday environment and its objects, linking the physical to the digital world and allowing people and "things" to be connected anytime, anywhere, with anything and anyone ideally using any network and service. IoT is regarded as a dynamic and global network of interconnected "things" uniquely addressable, based on standard and interoperable communication protocols and with self-configuring capabilities. Despite still being at an early development, adoption and implementation stage, Industry 4.0 and IoT can provide a multitude of contemporary solutions, applications and services. Hence, they can improve life quality and yield significant personal, professional and economic opportunities and benefits in the near future. This study scrutinizes IoT in the Industry 4.0 context. More specifically, it presents related studies, describes the IoT concept and explores some of the numerous IoT application domains. Moreover, it presents and analyzes the concept of Industry 4.0 and the benefits it offers as well as the relevant key technologies (e.g. industrial internet of things (IIoT), cyber-physical systems (CPSs), cloud computing, big data and advanced data analytics). Furthermore, it describes the concept of intelligent manufacturing and highlights the main IoT and Industry 4.0 challenges and open research issues. Finally, the need for innovation in the industrial domain and the impact and benefits that IoT and Industry 4.0 provide to everyday life and industries is described.
\end{abstract}

KEYWORDS: Internet of Things, Industry 4.0, IoT applications, Industrial Internet of Things, Ubiquitous computing, Cyber-physical systems.

JEL CLASSIFICATION: O14, O30, L86, L80, L60, L00.

DOI: $10.2478 /$ IJEK-2019-0001

Received: September $9^{\text {th }}, 2018$

1st Revision: May 22, 2019

Accepted: May 31 ${ }^{\text {st }}, 2019$

Reference: Lampropoulos, G., Siakas, K., Anastasiadis, T. (2019). Internet of Things in the Context of Industry 4.0: An Overview. International Journal of Entrepreneurial Knowledge, 7(1), 4-19. doi: 10.2478/ijek-2019-0001

\section{INTRODUCTION}


Nowadays, due to the rapidly evolving technological advancements, smart devices are able to interconnect, communicate and interact over the Internet. Moreover, over the years, the size of these devices has been reduced whereas their processing power and storage capabilities have significantly increased. Contemporary, smart devices are equipped with advanced embedded systems and have the capabilities of communication, sensing, actuation as well as real-time data retrieval, collection, storage and processing. Internet of Things (IoT) is a rapidly growing innovative technology with various applications, functions and services in everyday life and in a wide variety of domains. IoT aims at pervading our everyday environment and its objects, linking the physical world to the digital world and allowing "people and devices to be connected anytime, anywhere, with anything and with anyone" (Vermesan et al., 2011; Perera et al., 2013). IoT and more specifically Industrial Internet of Things (IIoT) along with the digitalization and automation of industrial manufacturing are thought to be what truly have initiated the fourth industrial revolution (Industry 4.0). Industry 4.0 combines traditional manufacturing and industrial practices with innovative technologies, such as large-scale machine-to-machine (M2M) communications, IoT, cyber-physical systems (CPSs), etc. Moreover, it aims at fundamentally transforming traditional industries into intelligent ones by introducing self-maintainability, selfoptimization, self-cognition, and self-customization into the industry. There is no doubt that by utilizing the advanced and dynamic network of interconnected devices, IoT can offer a magnitude of solutions to industries as well as multitude of contemporary and advanced applications and services that cannot only enhance life quality, but can also lead to personal, professional and economic opportunities and benefits (Li et al., 2011).

This study involves the analysis of IoT in the context of Industry 4.0. More specifically, it presents related studies (Section 1) and describes the concept of IoT (Section 2). Additionally, it explores some of the numerous IoT application domains (e.g. healthcare and sanitary, smart cities, industrial, smart environments, etc.) (Section 3) and presents the context of Industry 4.0 and the benefits it offers as well as the relevant key technologies, that is, industrial internet of things (IIoT), cyber-physical systems (CPSs), big data and advanced data analytics as well as cloud computing (Section 4). Furthermore, the concept of intelligent manufacturing is described (Section 5) and the main Io'T and Industry 4.0 challenges and open research issues are highlighted (Section 6). Finally, the main viewpoints of this study as well as the need for innovation in the industrial domain and the impact and benefits that IoT and Industry 4.0 provide to everyday life and industries are presented and described (Section 7).

\section{RELATED WORK}

Many elaborate studies which present and analyze the concept of IoT, its characteristics, application domains and key enabling technologies have been conducted. Atzori et al. (2010) conducted a survey in reference to IoT. They elaborated on different IoT visions and paradigms and reviewed key enabling technologies. Moreover, IoT application domains were analyzed and grouped into transportation and logistics, healthcare, personal and social, smart environment and futuristic domains. Finally, relevant open issues and challenges such as standardization activities, addressing and networking, security and privacy were examined (Atzori et al., 2010). Sundmaeker et al. (2010) presented the main vision and challenges for realizing IoT. More specifically, they analyzed the concept of IoT, its application domains as well as its supporting and enabling key technologies (Sundmaeker et al., 2010). In their study, Gubbi et al. (2013) presented a "Cloud" based centric vision for worldwide implementation of IoT. They also discussed the overall IoT vision as well as key enabling technologies and application domains along with some IoT trends and taxonomy. Moreover, they went through open challenges and future trends in IoT and they presented a case study of data analytics on the Aneka/Azure cloud platform. Finally, the need for convergence of wireless sensor network (WSN), the Internet and distributed computing was emphasized (Gubbi et al., 2013). Al-Fuqaha et al. (2015) carried out a study in which they focused on technical details that involve enabling technologies, the most relevant protocols (application, service discovery infrastructure etc.) and key IoT issues and challenges. A 5-layer model of IoT architecture comprising objects, object abstraction, service management, application and 
business layers was presented. Furthermore, identification, sensing, communication, computation, services and semantics, which are the six main elements vital to promote IoT functionality, were analyzed (Al-Fuqaha et al., 2015). With the aim of emphasizing the need for advanced IoT middleware, Ngu et al. (2016) conducted a survey in reference to the capabilities, the issues and the enabling technologies of the existing IoT middleware. State-of-the-art middleware solutions to realizing IoT applications and a thorough analysis of the challenges and the enabling technologies in developing an IoT middleware were presented. Moreover, they classified the different architecture types of IoT middleware, they assessed and outlined relevant key research challenges and finally, they presented a comparative analysis of emerging IoT middleware systems (Ngu et al., 2016). In their study, Pereira \& Romero (2017) presented a comprehensive definition about the concept of Industry 4.0 and provided several viewpoints regarding its vision. In addition, they described the key Industry 4.0 technology enablers and analyzed the impact and influence of this new industrial paradigm (Pereira \& Romero, 2017).

\section{INTERNET OF THINGS}

According to Atzori et al. (2010), the semantic definition of IoT could be determined as " $a$ world-wide network of interconnected objects uniquely addressable, based on standard communication protocols" (Atzori et al., 2010). Moreover, based on Vermesan et al. (2011), IoT can be regarded as a dynamic and global network infrastructure based on standard and interoperable communication protocols and with selfconfiguring capabilities. In this specific network infrastructure, physical and virtual "things" are interconnected, "have identities, physical attributes, and virtual personalities and use intelligent interfaces, and are seamlessly integrated into the information network" (Vermesan et al., 2011).

In the context of IoT, this new dimension of "things" can be considered as an extension of the existing human computer interaction and communication. More specifically, any physical or virtual entity, which "exists and moves in space and time and is capable of being identified either by assigned identification numbers, names and/or location addresses" and of being integrated into communication networks, can be regarded as a "thing" (Vermesan et al., 2011). Moreover, "things" become active participants and context aware, react autonomously and create services without the necessity of direct human intervention. IoT will provide a symbiotic interaction between the physical and virtual worlds and allow people and "things" to be connected anytime, anyplace, with anything and anyone, ideally using any path/network and any service (Atzori et al., 2010; Vermesan et al., 2011).

IoT aims at implementing autonomous, robust and secure connections and data exchange between devices and real-world applications. In addition, it contributes to the fulfillment of M2M communication and interconnectivity as well as to the integration of intelligence into devices. Thus, devices will be able to process information and data and make real-time autonomous human like intelligent decisions without requiring any human involvement and/or intervention (Khan et al., 2012). Hence, it can contribute to creating a potentially better world for people in which the "things" around them know what they like, what they want and what they need and act accordingly, without having to take explicit instructions from humans (Perera et al., 2014).

\section{APPLICATION DOMAINS OF INTERNET OF THINGS}

Io' $\mathrm{T}$ is considered to be a rapidly growing innovative technology with various applications, functions and services in everyday life and in a wide range of markets and industries. Although the recent advances in ubiquitous computing and the potentialities offered by the IoT render the development of a tremendous number of applications feasible, only a few are currently available (Atzori et al., 2010). IoT applications aim at improving life quality for the end-user community and supporting infrastructure and general-purpose operations (Akpakwu et al., 2017). Hereby, while being designed, the 
complexity and the scale of the problem to be solved as well as the particularities, requirements and characteristics of the specific domains in which they will be implemented should be taken into consideration as there is no one-fits-for-all solution. Moreover, they should be designed cautiously so as to satisfy multiple objectives and requirements while simultaneously increasing the quality of experience (QoE) and quality of service (QoS) levels.

Io'T solutions can be applied to numerous domains and environments hence they can be grouped into application domains in various ways. Atzori et al. (2010) conducted a survey in which they categorized IoT applications in the following domains (Atzori et al., 2010):

- Transportation and logistics domain: Applications of this domain involve logistics, assisted driving, mobile ticketing, environment monitoring, augmented maps etc.

- Healthcare domain: Applications of this domain involve tracking, identification, authentication, data collection, sensing etc.

- Smart environment domain: Applications of this domain involve comfortable homes/offices, industrial plants, smart museum and gym etc.

- Personal and social domain: Applications of this domain involve social networking, historical queries, losses and thefts etc.

- Futuristic domain: Applications of this domain involve robot taxi, city information model, enhanced game rooms etc.

Gubbi et al. (2013) classified IoT applications into the following application domains: 1) personal and home, 2) enterprise, 3) utilities and 4) mobile, taking into consideration the impact, type of network availability, coverage, scale, heterogeneity, repeatability and user involvement (Gubbi et al., 2013). While, in their study, Sundmaeker et al. (2010) grouped IoT applications into the following three application domains (Sundmaeker et al., 2010):

- Environmental domain: It involves applications that protect, monitor and develop all natural resources, environmental management services, energy management, recycling, agriculture etc.

- Industrial domain: Applications of this domain involve financial or commercial transactions between enterprises, organizations and other entities. Moreover, they refer to manufacturing, logistics, banking, financial governmental authorities etc.

- Social domain: It involves applications with regard to the development and inclusion societies, cities, and people as well as governmental services towards citizens and other society structures etc.

A number of IoT applications are presented below based on (Atzori et al., 2010; Sundmaeker et al., 2010; Vermesan et al., 2011; Khan et al., 2012; Miorandi et al., 2012; Gubbi et al., 2013; Borgia, 2014; Xu et al., 2014; Zanella et al., 2014; Perera et al., 2015; Gilchrist, 2016):

\subsection{Healthcare and sanitary}

The medical domain is one of the first industries which adopted IoT and was heavily influenced. IoT technologies can create new opportunities, services and applications so as to improve the healthcare and sanitary domain. Moreover, IoT platforms and services enhance current living solutions and facilitate the realization of Ubiquitous healthcare vision that is "bealthcare to anyone, anytime, and anywhere by removing location, time and other restraints while increasing both the coverage and quality of healthcare" (Lee \& Shim, 2009). Furthermore, in the context of IoT, all objects in the healthcare industry will be equipped with sensors and patients will carry mobile medical sensors. Hence, through the interconnection of these heterogeneous sensors, objects and patients will be able to be remotely traced and monitored in real time. This fact will simplify and automate the process of collecting data thus generating an insurmountable quantity of data that can be utilized for further scientific and medical studies. With a view to preventing the onset of health problems more efficiently, IoT technologies improve patient treatment and well-being and personalize QoE and QoS. Moreover, they optimize and enhance 
workflow, operations and drug management while simultaneously decrease operational costs. They also provide more effective methods for medical records and data analysis as well as ad hoc diagnosis by taking advantage of rapidly automated decisions and continuous advanced remote patient state monitoring. Finally, by offering a means of automation, supervision and communication for remote outpatients, IoT has an enormous impact on independent living and provides smart solutions for ameliorating life quality and supporting the aging population.

\subsection{Smart cities}

The rapid urban growth is already placing a considerable strain on the existing infrastructure and utilities and highlighting the need for more sustainable urban planning and public services. IoT applications and services are being exploited in order for these new requirements to be satisfied and the societal changes commensurate with this rapid growth to be responded to. Moreover, autonomic city and home networks will be intelligent and capable of sensing, monitoring and adapting to environmental changes as well as reacting to human activities. More specifically, through IoT, smart technologies and devices are interconnected and as a result, they are able to improve and enhance the quality and lifestyle for city dwellers as well as ensure that their essential services are provided. Furthermore, through IoT every aspect of urban life can be improved by creating smart cities that infuse the already established city services and utilities that residents interact with on a daily basis, optimizing the usage of city infrastructure, resources and facilities and enhancing city dwellers' life quality. Some of the many benefits of utilizing IoT applications and services in smart cities involve:

- Energy efficient utilities, buildings and facilities that will reduce the long-term costs and waste.

- Novel and effective systems of consuming, managing, distributing and renewing resources.

- Advanced traffic control and monitoring systems as well as more reliable public transportation means and pedestrian support.

- Advanced monitoring and security surveillance systems so as to improve public safety and security.

\subsection{Smart environments}

By utilizing fully interconnected technological devices and embedded systems, IoT aims at pervading our everyday environment and its objects and creating new ways to interact with these smart environments. The concept of smart environments evolves from ubiquitous computing and promotes the idea of "a physical world that is richly and invisibly interwoven with sensors, actuators, displays, and computational elements, embedded seamlessly in the everyday objects of our lives, and connected through a continuous network" (Weiser et al., 1999). Moreover, Cook \& Das (2005) described smart environments as "a small world where all kinds of smart devices are continuously working to make inhabitants' lives more comfortable". Additionally, they defined that "smart" refers to the ability to autonomously acquire and apply knowledge, while "environment" refers to our surroundings (Cook \& Das, 2005).

Through using IoT in combination with automated software agents for real time tracking and monitoring, smart environments become a technological ecosystem of various interconnected devices. These smart devices can securely communicate and interact as well as retrieve, process, store and exchange data in real time. By integrating these heterogeneous data into applications, the adaptation process to dwellers' and environmental continuously changing needs is facilitated. As a result, their requirements are being promptly and satisfactorily met. Moreover, IoT applications in this domain aim at improving the current environmental safety by reducing and mitigating the potential impact of damage and disaster. Io'T technologies allow the development of innovative real-time monitoring and decision-making support systems and applications regarding environmental issues, such as early prediction and detection of natural disasters, weather conditions etc. 


\subsection{Transportation and logistics}

Nowadays, vehicles as well as roads and transported goods, are equipped with more sophisticated technological devices such as on near field communication (NFC) tags, radio-frequency identification (RFID) tags, actuators, sensors etc. IoT technologies can be used to enhance the potential of these systems and optimize their use in the domains of transportation, logistics and suppliers, which are regarded as essential components to the productivity of many industries. Intelligent transportation systems (ITS) are able to communicate, share and exchange mission-critical information and data promptly, timely and accurately. Hence, they are used to ensure that the transportation network is efficiently monitored and controlled.

Io'T offers several contemporary applications and services and in combination with the ubiquitous $5 \mathrm{G}$ mobile networks can provide industries with intelligent transportation and logistics systems. These systems provide solutions which are designed specifically for certain needs and goals, thus accelerating productivity, profitability and operations. Moreover, they offer real-time monitoring and tracking throughout the entire supply chain, thus helping enterprises increase end-to-end visibility as well as maintain efficient transportation control and cost-effective management. In addition, they conduct more effective route planning and optimization, allow for better energy efficiency and reduce the overall system downtime.

\subsection{Industry}

A specific category of Io'T focuses on its applications and use cases in modern industries and intelligent manufacturing is Industrial Internet of Things (IIoT). It is considered to be a complex system of a wide variety of systems. Moreover, it comprises a key component to the industrial domain and is closely related to the fourth industrial revolution (Industry 4.0). It combines several innovative key technologies so as to produce a system that functions more effectively than the sum of its parts. This specific domain is characterized by its diverse innovative applications and services, its various interconnected devices as well as its novel manufacturing operations and is analyzed in detail in Section 4.1.1.

\section{INDUSTRY 4.0 AND THE INVOLVED KEY TECHNOLOGIES}

Over the last years, the global industrial landscape has drastically changed due to successive technological advancements, developments and innovations. The fourth industrial revolution (Industry $4.0)$ is characterized by its diverse innovative applications and services, its various interconnected devices as well as its novel manufacturing operations (Lampropoulos et al., 2018). Industry 4.0 can be regarded as a highly integrated, digitalized, automated and autonomous, and efficient manufacturing environment. Wee et al. (2015) defined Industry 4.0 as the "digitization of the manufacturing sector, with embedded sensors in virtually all product components and manufacturing equipment, ubiquitous cyber-physical systems, and analysis of all relevant data". In addition, they quoted that Industry 4.0 is driven by the following four technological clusters: 1) data, computational power and connectivity, 2) analytics and intelligence, 3) human-machine interaction and 4) digital-to-physical conversion (Wee et al., 2015). Furthermore, according to Schmidt et al. (2015), Industry 4.0 combines the powers of traditional industries with cutting edge technologies enabling smart products to be integrated into intertwined digital and physical processes. These processes interact with each other and cross geographical and organizational borders (Schmidt et al., 2015). As reported by Geissbauer et al. (2016), Industry 4.0 focuses on the end-to-end digitalization of all physical assets and their integration into digital ecosystems, while enabling them to seamlessly generate, analyze and communicate data. Additionally, they stated that 1) the digitalization and integration of vertical and horizontal value chains, 2) the digitalization of product and service offerings and 3) the digital business models and customer access are the leading driving factors of Industry 4.0 (Geissbauer et al., 2016). Based on Koch et al. (2014), Industry 4.0 constitutes a new level 
of organization and control throughout the entire value chain of the life cycle of products. Moreover, it is geared towards increasingly personalized customer requirements and enhances availability of all relevant information in real time by connecting all involved people, things and systems. Thus, it creates "a dynamic self-organizing, real-time optimized value-added connections within and across companies" (Koch et al., 2014). According to Hermann et al. (2016), the main design principles of the various Industry 4.0 components are: 1) interoperability, 2) virtualization, 3) decentralization, 4) real-time capability, 5) service orientation and 6) modularity (Hermann et al., 2016). Moreover, based on Pfohl et al. (2015), the main characterizing features of Industry 4.0 are: 1) digitalization, 2) autonomization, 3) transparency, 4) mobility, 5) modularization, 6) network-collaboration, 7) socializing (Pfohl et al., 2015). Additionally, Zhou et al. (2015) quoted that the following eight planning objectives are the prerequisites for achieving Industry 4.0 are: 1) standardization of systems, 2) efficient management, 3) establishment of a comprehensive and reliable industrial broadband infrastructure, 4) safety and security, 5) organization and design of work, 6) staff training and continuing professional development, 7) establishing a regulatory framework and 8) improving the efficiency of resource use (Zhou et al., 2015).

In accordance with MacDougall (2014), Industry 4.0 represents a paradigm shift from "centralized" to "decentralized". By decentralizing intelligence, intelligent object networking and independent process management are developed. A crucial new aspect of the manufacturing and production process is the dynamic interaction between the real and virtual worlds in which the industrial production machinery no longer simply processes the products, but it also communicates with them. As a result, industry, production value chains and business models are radically transformed into intelligent ones, thus leading to the development of smart factories (MacDougall, 2014). Moreover, according to (Pereira \& Romero, 2017), this new manufacturing paradigm lays high emphasis on creating smart products and developing smart processes by utilizing smart machines and transforming conventional manufacturing systems in smart factories. Smart factories allow the development of intelligent manufacturing environments throughout the entire value chain and they are the outcome of integration through digitalization, usage of flexible and adaptive structures and strategies as well as of artificial intelligence methods (Hajrizi, 2016; Pereira \& Romero, 2017).

\subsection{Key technologies involved in Industry 4.0}

Industry 4.0 aims at enhancing and upgrading the current manufacturing facilities, management and maintenance systems and technologies to an intelligent level by utilizing key technologies such as IoT, Internet of Services (IoS), CPSs, autonomous, flexible and cooperative robotics, simulations that leverage real-time data and mirror real world into a virtual model, big data analytics, augmented reality (AR), additive manufacturing, information and communication technologies (ICT) and advanced networking technologies (e.g. cloud computing etc.) (Lasi et al., 2014; Lee et al., 2015; Pereira \& Romero, 2017; Zhong et al., 2017). Moreover, it seeks to address the dynamic global market and the competitive nature of today's industries in line with the continuously changing customers' and market needs. Horizontal and vertical system integration will allow that capabilities, functions, departments and enterprises evolve in an interconnected network that enables an automated value chain. According to Zhong et al. (2017), intelligent manufacturing will pave the way for the advancement of modern industry and economy as it will apply cutting-edge technologies to various traditional products and systems (Zhong et al., 2017). In an Industry 4.0 context, data collection, analysis and comprehension from many diverse sources, including production systems and equipment, as well as customer management enterprise systems, will become the norm to support decision making in real time. Some of the key technologies involved in Industry 4.0 are briefly described and analyzed below:

\subsubsection{Industrial internet of things}

Industrial Internet of Things (IIoT) is a specific category of IoT which focuses on its applications and use cases in modern industries and intelligent manufacturing. IIoT, which is used in the context of Industry 4.0, can be considered to be a complex system of diverse systems and devices. More 
specifically, with a view to producing a system which functions more efficiently than the sum of its parts, IIoT combines several contemporary key technologies (Lampropoulos et al., 2018).

Through the use of appropriate services, networking technologies, applications, sensors, software, middleware and storage systems, IIoT provides solutions and functions which develop insight and improve the potential and capability of monitoring and controlling enterprises processes and assets. IIoT services and applications provide vital solutions for more effective scheduling, planning and controlling of manufacturing operations and systems (Bi et al., 2014; Gilchrist, 2016). Additionally, through the various interconnected devices that are able to communicate and interact both with each other and with more centralized controllers, IIoT will decentralize analytics and decision-making, thus rendering real-time responses and reactions feasible (Şen et al., 2018). As a result, the overall availability and maintainability of enterprises is enhanced, their operational efficiency is improved, productivity is accelerated, their product time-to-market is decreased by reducing unplanned downtime and their overall operational efficiency is optimized accomplishing, thus, enormous potential for unprecedented levels of economic growth and productivity efficiency (Bi et al., 2014; Gilchrist, 2016).

\subsubsection{Cyber-physical systems}

Cyber-physical technologies and frameworks, also known as cyber-physical systems (CPSs), have been increasingly adopted in industry due to the significant technological advancements in the domains of computer science, ICT and manufacturing (Monostori, 2014; Lu \& Cecil, 2015; Mourtzis et al., 2016). Unlike traditional embedded systems, CPS-enabled systems contain "cybertwined services such as control algorithms and computational capacities" (Zhong et al., 2017) along with specialized computational capabilities, physical assets and networked interactions (Lee et al., 2015) and involve a large number of transdisciplinary methodologies. The CPSs concept facilitate an ecosystem of cyber manufacturing, where smart machines process production data through a wireless embedded network system. Moreover, CPSs are defined as transformative technologies that can seamlessly link the physical with the virtual world through their advanced and novel systems (Mourtzis et al., 2016). Hence, they are designed and developed to have both physical inputs and outputs so as to enable and enhance the interaction with humans using innovative modalities (Baheti \& Gill, 2011; Lu et al., 2017).

\subsubsection{Cloud computing}

Cloud computing or simply "Cloud" plays a leading role in enhancing and transforming the current industry as it is a kind of outsourcing that combines large numbers of compute servers and resources with a view to offering computer programs, high-level services and resources on an on-demand or payper-cycle basis in real time (Bhardwaj et al., 2010). According to Wang et al. (2010), cloud computing is "a set of network enabled services, providing scalable, QoS guaranteed, normally personalized, inexpensive computing infrastructure on demand, which could be accessed in a simple and pervasive way" (Wang et al., 2010). Cloud computing is divided into three levels of service offerings, namely Software as a Service (SaaS), Platform as a Service (PaaS) and Infrastructure as a Service (IaaS) which support different levels of virtualization and management of the solution stack (Bhardwaj et al., 2010).

The use of advanced applications and services that dynamically scale with the increased number of users is considered to be one of the main benefits of cloud computing (Lu \& Cecil, 2015). Moreover, users and enterprises have prompt access to applications, programs and services which are rapidly provisioned with minimal management effort and are hosted in the "Cloud" at any time and from any place. Hence, enterprises in the industrial domain use various cloud-based applications widely in order to enhance the crucial for their effective function systems, such as Customer Relationship Management (CRM), Human Resource Management (HRM) etc. Furthermore, enterprises that utilize cloud computing can avoid the complexity of owning and maintaining their own Information Technology (IT) infrastructure and the up-front costs which can be accomplished by a "pay-as-you-go" method (Lu \& Cecil, 2015) allowing, thus, the enterprises to start small and invest into more resources if there is 
more service demand (Zhang et al., 2010; Zhong et al., 2017). Some further benefits and significant advantages include high availability and maintainability, consistent accessibility to data and services from any connected device as well as reduced development cost and product time-to-market. Taking into consideration the continuously evolving nature, the numerous application domains and multiple benefits of cloud computing, a lot of profits can be yielded in the industrial domain, thus, more and more enterprises of different sizes and types are rapidly adopting this advanced technology with the aim of enhancing their capabilities and capacity at a minimum cost (Zhong et al., 2017).

\subsubsection{Big data and advanced data analytics}

The digitalization of everyday life through the adoption of smart devices and advanced technologies (e.g. IoT, Artificial Intelligence (AI), Social Networks (SNS) etc.) has led to the increase of data sources and the diversity of digital content as well as data types, forms and structures (Gahi et al., 2016). Consequently, an enormous volume of heterogeneous data, named big data, is generated and increases exponentially on a daily basis. Volume, variety, veracity, velocity and value are the key factors which characterize and differentiate big data from traditional data (McAfee \& Brynjolfsson, 2012; Gahi et al., 2016). Big data plays a key role in industries and intelligent manufacturing as it can provide enterprises with numerous advantages, merits and benefits through various predictive and prescriptive insights. Hence, enterprises, which want to remain competitive, should give priority to the implementation and utilization of contemporary advanced analytical tools, techniques, methods and applications with the aim of processing big data, gleaning intelligence and retrieving the value of the vital data in each case. These tools are named big data analytics (BDA) and use parallel and analytic techniques to analyze huge volume of diverse, rapidly transforming data enabling, thus, the collection, the process and the management of vital information and statistics (Gahi et al., 2016; Parwez et al., 2017). By far, the most effective way for enterprises to gain immense benefits over their competitors, optimize operations, enhance productivity, quality and efficiency and reduce operational costs is to use all the newly gained knowledge in order to generate invaluable insights and improve equipment service and maintainability (Gilchrist, 2016). Nonetheless, in order to fully utilize big data and exploit all of its benefits, enterprises must change their decision-making culture and take into consideration that no matter how much the potential of big data and analytic tools increase, the need for human insight should not be overlooked (McAfee \& Brynjolfsson, 2012).

\section{INTELLIGENT MANUFACTURING IN THE CONTEXT OF INDUSTRY 4.0}

Manufacturing comprises a core industrial component which has a vital impact on people's livelihood and a nation's economy. Additionally, it is one of the largest and highly interconnected IoT markets and it involves a wide variety of operations, processes, services, products etc. With the aim of enhancing the overall production, productivity and product quality management (PQM) throughout the various stages of the life-cycle of products, IoT offers applications and services which include advanced monitoring and tracking, performance and maintainability optimization and human machine interaction. Hence, it stands to reason that IoT can provide a lot of solutions to the manufacturing domain which is characterized by its complexity and breadth of applications, its diverse CPSs and its manufacturing operation management (MOM) methodologies (Zhong et al., 2017).

In the context of Industry 4.0, intelligent manufacturing, also known as smart manufacturing, uses Service-Oriented Architecture (SOA) and is considered to be a novel manufacturing model that takes advantage of and fully utilizes various advanced information and manufacturing techniques, methodologies and technologies (Zhong et al., 2017). It aims at fundamentally transforming traditional enterprises into intelligent ones so as to effectively respond to demand-dynamic economics keyed on "customers, partners and the public; enterprise performance and variability management; real-time integrated computational materials engineering and rapid qualification, demand-driven supply chain services; and broad-based workforce involvement' (Davis et al., 2012). Intelligent manufacturing uses the combined intelligence of 
people, processes and machines so as to increase production, product quality and productivity efficiency. It offers smart solutions for the detection and monitoring of potential damage, malfunctions and breakdowns. Moreover, it enhances control and management, improves maintainability and availability and optimizes resource management and sharing. Additionally, it applies cutting-edge technologies to various traditional systems, services and products (Zhong et al., 2017). As a result, it is obvious that intelligent manufacturing has a drastic impact on the overall function and economics state of enterprises and will pave the way for the advancement of modern industries.

Intelligent manufacturing aims at developing real-time, autonomous and human like intelligent decision-making systems that reduce the need for human involvement and intervention. In order for this to be accomplished, artificial intelligence, machine learning, genetic algorithms and other advanced technologies, methodologies and techniques are used. This fact comprises a major distinguishing factor between intelligent manufacturing and traditional manufacturing. Nonetheless, the goal of both manufacturing domains remains the same, that is, to satisfy customers' requirements and market needs as well as maximize profits while simultaneously minimizing possible cost and waste (Kumar, 2002).

\section{OPEN RESEARCH ISSUES AND CHALLENGES OF INTERNET OF THINGS AND INDUSTRY 4.0}

It is obvious that in the context of Industry 4.0, IoT can enhance and transform the current industries and yield a lot of benefits due to its advanced technologies, applications and services. It is also vital to point out that IoT not only aims at transforming industries and increasing their productivity but also at adding value to the core purpose of enterprises and mitigating the weaknesses caused by legacy systems. Hence, it should be compatible with existing devices, systems and infrastructure and be able to embed intelligence into them. As a result, enterprises that are undergoing digital transformation will be facilitated to adopt and implement Io $\mathrm{T}$ and exploit its numerous benefits and solutions without having to directly invest in totally brand-new equipment as cost might far outweigh the immediate benefits. Nonetheless, in order for Industry 4.0 to be fully implemented and for IoT to be adopted and fully utilized by industries and enterprises, a lot of challenges and open issues should be looked into and addressed.

\subsection{Challenges and open research issues regarding internet of things implementation}

Many elaborate studies, which analyze vital IoT challenges, integration and implementation problems and open research issues, have been conducted. More specifically, open research issues involving standardization activities, addressing and networking as well as security and privacy were analyzed by Atzori et al. (2010). Key IoT challenges, such as interoperability and standardization, data and information confidentiality, encryption and privacy, naming and identity management, Io'T greening as well as object and network security were described by Khan et al. (2012). Communication and identification technologies, distributed system technologies and intelligence and emphasized security issues such as data confidentiality, privacy and trust were some of the main research challenges that Miorandi et al. (2012) looked into. In their study, Gubbi et al. (2013) examined and analyzed open challenges such as secure reprogrammable networks and privacy, QoS, energy efficient sensing, architecture and protocols, Geographic Information System (GIS) based visualization, data mining and cloud computing (Gubbi et al., 2013). The open challenges and issues which Borgia (2014) went over were: object mobility, M2M communications, device and data management, network architecture and system design, addressing, naming and traffic characterization and security (Borgia, 2014). According to the study conducted by Perera et al. (2014), privacy and data analytics, interoperability on products and services as well as resources and energy management are considered to be key challenges. Key IoT challenges and QoS criteria such as availability and reliability, mobility, performance and management, scalability and interoperability as well as security and privacy were examined by Al-Fuqaha et al. (2015). Breivold \& Sandström (2015) described the management of fault tolerance, functional safety, latency 
and scalability of data, mixed criticality and scalable as well as secure real-time collaboration as key IIoT challenges (Breivold \& Sandström, 2015). Data management and mining, security and privacy were regarded as the main challenges which enterprises face in IoT development by Lee \& Lee (2015). Sadeghi et al. (2015) focused on security and privacy challenges in IIo'T and their vulnerability to a variety of cyberattacks (Sadeghi et al., 2015).

To sum up, based on the above mentioned studies, the most significant and common IoT challenges and open research issues which industries and enterprises should be aware of are:

- Availability, reliability, mobility and other QoS criteria;

- Security, privacy and confidentiality of data;

- Interoperability and scalability;

- Fault tolerance and functionality safety;

- Management of operations, resources, energy and data;

- Networking addressing and identification;

- Architecture, protocols and standardization activities.

\subsection{Challenges and open research issues regarding the implementation of Industry 4.0}

Many elaborate studies, regarding vital challenges, integration and implementation problems and open research issues of Industry 4.0, have been carried out. Wang et al. (2016) and Vaidya et al. (2018) defined several challenges and fundamental issues in various sections that occur throughout the implementation of Industry 4.0. The identified sections in their studies were: 1) intelligent decisionmaking and negotiation mechanism, 2) high speed industrial wireless network (IWN) protocols, 3) manufacturing specific big data and its analytic, 4) system modeling and analysis, 5) cyber and property security and 6) modularized and flexible physical artifacts, 7) Investment issues (Wang et al., 2016; Vaidya et al., 2018). Based on Zhou et al. (2015), some of science and technology challenges concerning the implementation of Industry 4.0 involve the development of smart devices, the construction of network environments, big data analysis and processing and digital production (Zhou et al., 2015). In his study, Schröder (2016) pointed out the lack of a digital strategy in line with resource scarcity as well as the lack of standards and poor data security as the main obstacles for the technological implementation of Industry 4.0 (Schröder, 2016). Küsters et al. (2017) claimed that some manufacturers and enterprises hesitate to implement Industry 4.0 due to certain concerns and barriers. These include uncertainties about financial benefits, lack of strategies of coordinating across different organizational units, missing talent, skills and capabilities, hesitation to go through radical transformation and concerns regarding the third-party providers' security (Küsters et al., 2017). According to Kagermann et al. (2013), the three greatest challenges connected with implementing Industry 4.0 are 1) standardization, 2) work organization and 3) product availability (Kagermann et al., 2013). Chen et al. (2017) quoted that there are still issues and challenges to be coped with in regard to equipment intelligent requirements, deep integration networks and knowledge-driven manufacturing (Chen et al., 2017). $\mathrm{Lu}$ (2017) regarded interoperability as the main open issue in Industry 4.0 and analyzed the main principles to ensure high accuracy and efficiency of processes, that is accessibility, multilingualism, security, privacy, subsidiarity, the use of open standards, open source software and multilateral solutions (Lu, 2017). Bauer et al. (2016) carried out a Global Expert Survey including 300 expert companies (with at least 50 employees), split evenly across the United States of America, Germany and Japan in both Industry 4.0 technology suppliers and manufacturers. The expectations and attitudes are positive and optimistic. In total $90 \%$ of the respondents believe their competitiveness will increase with Industry 4.0 or stay the same, $89 \%$ expect Industry 4.0 to impact their operational effectiveness, and $80 \%$ consider that Industry 4.0 will have an impact on their business model. Moreover, based on their survey the main challenges of Industry 4.0 are (Bauer et al., 2016):

- Lack of courage to push through the radical change needed for introducing Industry 4.0;

- Lack of necessary talents for making Industry 4.0 happen;

- Lack of a clear business case justifying investments in Industry 4.0 IT architecture; 
- Difficulty in coordinating actions across different organizational units, such as research \& development (R\&D), IT, manufacturing, sales, and finance departments, due to poor interaction between them;

- Uncertainty about insourcing versus outsourcing and lack of knowledge about service providers;

- Concerns about cybersecurity when involving third-party technology/software and implementation providers;

- Concerns about data ownership when working with third-party providers;

- Challenges with integrating data from disparate sources to enable Industry 4.0 applications.

\section{DISCUSSION}

Technological advances and digitalization of everyday life have led to the increase of rapidly changing customers' needs and requirements. Simultaneously, the fierce competition which prevails in global markets has drastically risen. As a consequence, the need for flexibility and real time response to these changes is becoming vital. Therefore, with a view to fulfilling and satisfying these new demands, staying ahead of their competitors, enhancing their product and service quality and raising their profits, enterprises opt to implement new technological means, practices and methodologies and seek for new innovative approaches to increase their productivity. According to Francis \& Bessant (2005) and Tidd et al., (2005), the four broad types of innovation that enterprises mostly target at are: 1) product innovation, 2) process innovation, 3) position innovation and 4) paradigm innovation (Francis \& Bessant, 2005; Tidd et al., 2005). Generally, innovations result in more changes such as manufacturing paradigm shifts, progress in technologies etc., create new opportunities and bring about new challenges.

In order for enterprises to sustain in the context of globalization, create new values and drive innovation to achieve more competitive success in their business, they should adapt to the digital transformation and the virtualization process. Moreover, they should incorporate innovation in their manufacturing process and integrate technological and managerial approaches so as to strengthen their overall competence.

Industry 4.0 is a response to the newly created challenges in a fast-changing and evolving environment. It is regarded as a highly integrated, digitalized, automated, autonomous and efficient intelligent manufacturing environment and it constitutes a new level of organization and control throughout the entire value chains. More specifically, it puts emphasis on the development of smart factories and intelligent manufacturing and aims to deal with customers' and market changing needs and transform conventional industries into intelligent ones. In order for this to be achieved, Industry 4.0 combines the powers of traditional industries with cutting edge technologies (e.g. IIoT, CPSs), cloud computing, big data and advanced data analytics etc.) enabling physical assets to be integrated into intertwined digital and physical processes. The main contribution to the realization of Industry 4.0 was made by IoT. It allows people and "things" to be connected anytime, anywhere, with anything and anyone ideally using any network and service. Additionally, IoT pursues to pervade our everyday environment and its objects, linking the physical to the digital world. IoT which is regarded as a dynamic and global network of uniquely addressable interconnected "things" aims at implementing autonomous, robust and secure connections. Moreover, IoT provides various applications, functions and services in everyday life and in a wide range of markets and industries.

By implementing and adapting to Industry 4.0 and Io'T technologies, unprecedented levels of economic growth and productivity efficiency can be achieved by enterprises, such as:

- Development of production systems which are characterized by interoperability, flexibility, adaptability, agility and proactivity. 
- Optimization and improvement of efficiency, speed and quality particularly in engineering, operation, administration and decision-making.

- Enhancement of overall application, services and system availability and maintainability.

- Acceleration of productivity and reduction of lead time resulting in decreasing time-to-market.

- Facilitation of the adaptation to individualized customer requirements and market demands.

- Improvement of monitoring and controlling enterprises processes and assets.

- Reduction of overall cost and waste.

- Decentralization and digitalization of production.

- Capability of robust, enterprise-wide data analytics.

Industry 4.0 and Io'T can provide a multitude of contemporary solutions, applications and services and can yield significant personal, professional and economic opportunities. Hence, enterprises and industries that are able to fully implement and adapt to Industry 4.0 and IoT will reap many benefits and profits and will be able to stay ahead of market competition. Nonetheless, IoT and Industy 4.0 are still at an early stage of development, adoption and implementation, therefore there are still various open issues and challenges that need to be addressed.

\section{CONCLUSION}

IoT is an innovative and rapidly growing technology which offers various novel applications, services and solutions and links the physical to the digital world. It also allows people and "things" to be connected anytime, anywhere, with anything and with anyone ideally using any path/network and any service. Moreover, it improves the quality for the end-user community and our lives in general and supports infrastructure and general-purpose operations. In addition, it aims at transforming the current industries into intelligent ones utilizing the dynamic network of interconnected devices. Enhancing their operation and functionality, increasing their productivity and reducing their costs and waste are some of the many benefits and profits that enterprises can gain by using IoT. Moreover, enterprises that fully adopt IoT will be ahead of their competitors, become more agile, adapt to the continuously changing market, create products of higher quality that satisfy customers' needs and requirements.

Moreover, in the context of Industry 4.0, IoT, and more specifically IIoT, can be utilized in combination with other innovative technologies such as big data, cloud computing, CPSs etc. in order to enhance and transform the current manufacturing systems into intelligent ones. Industry 4.0 allows for machines to become independent entities that are able to collect and analyze data and give advice upon it without requiring any human intervention as it introduces self-maintainability, selfoptimization, self-cognition, and self-customization as well as intelligence to the industry. It seeks to cope successfully with the global competitive nature of today's markets and industries in line with the ever-changing customers' needs and requirements.

Although IoT offers a magnitude of solutions to industries as well as multitude of contemporary and advanced applications and services, it is still at an early stage of development, adoption and implementation. Thus, in order for the various current challenges and open issues to be encountered and solved, further research should be carried out. All in all, the complete implementation and prompt adoption of IoT along with appropriate utilization of its novel technologies, applications and services cannot only improve life quality, but can also yield significant personal, professional and economic opportunities and benefits in the near future. 


\section{REFERENCES}

Akpakwu, G. A., Silva, B. J., Hancke, G. P., \& Abu-Mahfouz, A. M. (2017). A survey on 5G networks for the Internet of Things: Communication technologies and challenges. IEEE Access, 6, 36193647.

Al-Fuqaha, A., Guizani, M., Mohammadi, M., Aledhari, M., \& Ayyash, M. (2015). Internet of things: A survey on enabling technologies, protocols, and applications. IEEE communications surveys \& tutorials, 17(4), 2347-2376.

Atzori, L., Iera, A., \& Morabito, G. (2010). The internet of things: A survey. Computer networks, 54(15), 2787-2805.

Baheti, R., \& Gill, H. (2011). Cyber-physical systems. The Impact of Control Technology, T. Samad and AM Annaswamy. IEEE Control Systems Society, 1.

Bauer, H., Baur, C., Mohr, D., Tschiesner, A., Weskamp, T., Alicke, K., \& Wee, D. (2016). Industry 4.0 after the initial hype-Where manufacturers are finding value and how they can best capture it. McKinsey Digital.

Bhardwaj, S., Jain, L., \& Jain, S. (2010). Cloud computing: A study of infrastructure as a service (IAAS). International Journal of engineering and information Technology, 2(1), 60-63.

Bi, Z., Da Xu, L., \& Wang, C. (2014). Internet of things for enterprise systems of modern manufacturing. IEEE Transactions on industrial informatics, 10(2), 1537-1546.

Borgia, E. (2014). The Internet of Things vision: Key features, applications and open issues. Computer Communications, 54, 1-31.

Breivold, H. P., \& Sandström, K. (2015, December). Internet of things for industrial automation-challenges and technical solutions. In 2015 IEEE International Conference on Data Science and Data Intensive Systems (pp. 532-539). IEEE.

Chen, B., Wan, J., Shu, L., Li, P., Mukherjee, M., \& Yin, B. (2017). Smart factory of industry 4.0: Key technologies, application case, and challenges. IEEE Access, 6, 6505-6519.

Cook, D., \& Das, S. K. (2004). Smart environments: Technology, protocols and applications (Vol. 43). John Wiley \& Sons.

Da Xu, L., He, W., \& Li, S. (2014). Internet of things in industries: A survey. IEEE Transactions on industrial informatics, 10(4), 2233-2243.

Davis, J., Edgar, T., Porter, J., Bernaden, J., \& Sarli, M. (2012). Smart manufacturing, manufacturing intelligence and demand-dynamic performance. Computers \& Chemical Engineering, 47, 145-156.

Francis, D., \& Bessant, J. (2005). Targeting innovation and implications for capability development. Technovation, 25(3), 171-183.

Gahi, Y., Guennoun, M., \& Mouftah, H. T. (2016, June). Big data analytics: Security and privacy challenges. In 2016 IEEE Symposium on Computers and Communication (ISCC) (pp. 952-957). IEEE.

Geissbauer, R., Vedso, J., \& Schrauf, S. (2016). Industry 4.0: Building the digital enterprise. Retrieved from: bttps:// www.pwc.com/gx/en/industries/industries-4.0/landing-page/industry-4.0-building-your-digitalenterprise-april-2016.pdf.

Gilchrist, A. (2016). Industry 4.0: the industrial internet of things. New York, NY: Apress., ISBN: 1484220463.

Gubbi, J., Buyya, R., Marusic, S., \& Palaniswami, M. (2013). Internet of Things (IoT): A vision, architectural elements, and future directions. Future generation computer systems, 29(7), 1645-1660.

Hajrizi, E. (2016). Smart solution for smart factory. IF AC-PapersOnLine, 49(29), 1-5.

Hermann, M., Pentek, T., \& Otto, B. (2016, January). Design principles for industrie 4.0 scenarios. In 201649 th Hawaii international conference on system sciences (HICSS) (pp. 3928-3937). IEEE.

Kagermann, H., Helbig, J., Hellinger, A., \& Wahlster, W. (2013). Recommendations for implementing the strategic initiative INDUSTRIE 4.0: Securing the future of German manufacturing industry; final report of the Industrie 4.0 Working Group. Forschungsunion.

Khan, R., Khan, S. U., Zaheer, R., \& Khan, S. (2012, December). Future internet: the internet of things architecture, possible applications and key challenges. In 2012 10th international conference on frontiers of information technology (pp. 257-260). IEEE. 
Koch, V., Kuge, S., Geissbauer, R., \& Schrauf, S. (2014). Industry 4.0: Opportunities and challenges of the industrial internet. Strategy \& $P w C$.

Kumar, S. (2002). Intelligent Manufacturing Systems. B.I.T. Mesra, Ranchi, India. Retrieved from https://pdfs.semanticscholar.org/fc60/02a47a1a9b312bda871b47569edeeb8545f9.pdf

Küsters, D., Praß, N., \& Gloy, Y. S. (2017). Textile Learning Factory 4.0-Preparing Germany's Textile Industry for the Digital Future. Procedia Manufacturing, 9, 214-221.

Lampropoulos, G., Siakas, K., \& Anastasiadis, T. (2018). Internet of Things (IoT) in Industry: Contemporary Application Domains, Innovative Technologies and Intelligent Manufacturing. International Journal of Advances in Scientific Research and Engineering, 4(10), 109-118.

Lasi, H., Fettke, P., Kemper, H. G., Feld, T., \& Hoffmann, M. (2014). Industry 4.0. Business \& information systems engineering, 6(4), 239-242.

Lee, C. P., \& Shim, J. P. (2009). Ubiquitous healthcare: Radio frequency identification (RFID) in hospitals. In Handbook of research on distributed medical informatics and e-health (pp. 273-281). IGI Global.

Lee, I., \& Lee, K. (2015). The Internet of Things (IoT): Applications, investments, and challenges for enterprises. Business Horizons, 58(4), 431-440.

Lee, J., Bagheri, B., \& Kao, H. A. (2015). A cyber-physical systems architecture for industry 4.0-based manufacturing systems. Manufacturing letters, 3, 18-23.

Li, J., Huang, Z., \& Wang, X. (2011, May). Notice of Retraction Countermeasure research about developing Internet of Things economy: A case of Hangzhou city. In 2011 International Conference on E-Business and E-Government (ICEE) (pp. 1-5). IEEE.

Lu, S., Xu, C., Zhong, R. Y., \& Wang, L. (2017). A RFID-enabled positioning system in automated guided vehicle for smart factories. Journal of Manufacturing Systems, 44, 179-190.

Lu, Y. (2017). Industry 4.0: A survey on technologies, applications and open research issues. Journal of Industrial Information Integration, 6, 1-10.

Lu, Y., \& Cecil, J. (2016). An Internet of Things (Io'T)-based collaborative framework for advanced manufacturing. The International Journal of Advanced Manufacturing Technology, 84(5-8), 1141-1152.

MacDougall, W. (2014). Industrie 4.0: Smart manufacturing for the future. Germany Trade \& Invest.

McAfee, A., Brynjolfsson, E., Davenport, T. H., Patil, D. J., \& Barton, D. (2012). Big data: the management revolution. Harvard business review, 90(10), 60-68.

Miorandi, D., Sicari, S., De Pellegrini, F., \& Chlamtac, I. (2012). Internet of things: Vision, applications and research challenges. Ad hoc networks, 10(7), 1497-1516.

Monostori, L. (2014). Cyber-physical production systems: Roots, expectations and R\&D challenges. Procedia Cirp, 17, 9-13.

Mourtzis, D., Vlachou, E., \& Milas, N. (2016). Industrial Big Data as a result of IoT adoption in manufacturing. Procedia Cirp, 55, 290-295.

Ngu, A. H., Gutierrez, M., Metsis, V., Nepal, S., \& Sheng, Q. Z. (2016). IoT middleware: A survey on issues and enabling technologies. IEEE Internet of Things Journal, 4(1), 1-20.

Parwez, M. S., Rawat, D. B., \& Garuba, M. (2017). Big data analytics for user-activity analysis and useranomaly detection in mobile wireless network. IEEE Transactions on Industrial Informatics, 13(4), 2058-2065.

Pereira, A. C., \& Romero, F. (2017). A review of the meanings and the implications of the Industry 4.0 concept. Procedia Manufacturing, 13, 1206-1214.

Perera, C., Liu, C. H., \& Jayawardena, S. (2015). The emerging internet of things marketplace from an industrial perspective: A survey. IEEE Transactions on Emerging Topics in Computing, 3(4), 585-598.

Perera, C., Liu, C. H., Jayawardena, S., \& Chen, M. (2014). A survey on internet of things from industrial market perspective. IEEE Access, 2, 1660-1679.

Perera, C., Zaslavsky, A., Christen, P., \& Georgakopoulos, D. (2013). Context aware computing for the internet of things: A survey. IEEE communications surveys \& tutorials, 16(1), 414-454.

Pfohl, H. C., Yahsi, B., \& Kurnaz, T. (2015). The impact of industry 4.0 on the supply chain. Iin Proceedings of the Hamburg International Conference of Logistics (HICL) 21: Sustainability in Logistics and Supply Chain Management. New Designs and strategies, eds W. Kersten, T. Blecker, and C. M. Ringle (Berlin: epubli GmbH), 31-58. 
Sadeghi, A. R., Wachsmann, C., \& Waidner, M. (2015, June). Security and privacy challenges in industrial internet of things. In 2015 52nd ACM/EDAC/IEEE Design Automation Conference (DAC) (pp. 1-6). IEEE.

Schmidt, R., Möhring, M., Härting, R. C., Reichstein, C., Neumaier, P., \& Jozinović, P. (2015, June). Industry 4.0-potentials for creating smart products: empirical research results. In International Conference on Business Information Systems (pp. 16-27). Springer, Cham.

Schröder, C. (2016). The challenges of industry 4.0 for small and medium-sized enterprises. FriedrichEbert-Stiftung: Bonn, Germany.

Şen, K. Ö., Durakbasa, M. N., Baysal, M. V., Şen, G., \& Baş, G. (2018). Smart Factories: A Review of Situation, and Recommendations to Accelerate the Evolution Process. In The International Symposium for Production Research (pp. 464-479). Springer, Cham.

Sundmaeker, H., Guillemin, P., Friess, P., \& Woelfflé, S. (2010). Vision and challenges for realising the Internet of Things. Cluster of European Research Projects on the Internet of Things, European Commision, 3(3), 34-36.

Tidd, J., Bessant, J., \& Pavitt, K. (2005). Managing innovation integrating technological, market and organizational change. John Wiley and Sons Ltd.

Vaidya, S., Ambad, P., \& Bhosle, S. (2018). Industry 4.0-a glimpse. Procedia Manufacturing, 20, 233-238.

Vermesan, O., Friess, P., Guillemin, P., Gusmeroli, S., Sundmaeker, H., Bassi, A., ... \& Doody, P. (2011). Internet of things strategic research roadmap. Internet of things-global technological and societal trends, 1(2011), 9-52.

Wang, L., Von Laszewski, G., Younge, A., He, X., Kunze, M., Tao, J., \& Fu, C. (2010). Cloud computing: a perspective study. New Generation Computing, 28(2), 137-146.

Wang, S., Wan, J., Li, D., \& Zhang, C. (2016). Implementing smart factory of industrie 4.0: an outlook. International Journal of Distributed Sensor Networks, 12(1), 3159805.

Weiser, M., Gold, R., \& Brown, J. S. (1999). The origins of ubiquitous computing research at PARC in the late 1980s. IBM systems journal, 38(4), 693-696.

Wee, D., Kelly, R., Cattel, J., \& Breunig, M. (2015). Industry 4.0-how to navigate digitization of the manufacturing sector. McKinsey \& Company, 58.

Zanella, A., Bui, N., Castellani, A., Vangelista, L., \& Zorzi, M. (2014). Internet of things for smart cities. IEEE Internet of Things journal, 1(1), 22-32.

Zhang, Q., Cheng, L., \& Boutaba, R. (2010). Cloud computing: state-of-the-art and research challenges. Journal of internet services and applications, 1(1), 7-18.

Zhong, R. Y., Xu, X., Klotz, E., \& Newman, S. T. (2017). Intelligent manufacturing in the context of industry 4.0: a review. Engineering, 3(5), 616-630.

Zhou, K., Liu, T., \& Zhou, L. (2015). Industry 4.0: Towards future industrial opportunities and challenges. In 2015 12th International Conference on Fuz:y Systems and Knowledge Discovery (FSKD) (pp. 2147-2152). IEEE. 